\title{
An unusual complication of the treatment of spontaneous pneumothorax
}

\author{
W. C. BARNSLEY AND M. WALTON \\ From Poole Hospital, Nunthorpe, Middlesbrough
}

\begin{abstract}
A 65-year-old painter was referred to the mass $x$ ray unit on 21 May 1963 because of dyspnoea, and the small film was reported as showing a right spontaneous pneumothorax with complete lung collapse and some mediastinal shift to the left. He was seen at the chest clinic on May 23 and, although very deaf, it was possible to elicit the history of many years of chronic cough with sputum. One week previously, on coming down the stairs in the morning, he felt a sudden stabbing pain over the sternum with breathlessness on slight exertion which had persisted. He was admitted to hospital on May 24.
\end{abstract}

On examination his general condition was good. He was dyspnoeic at rest with a degree of cyanosis, and he was restless; the apex beat was in the left anterior axillary line and there was absence of air entry over the right lung. His blood pressure was $150 / 90 \mathrm{~mm}$. Hg. It was decided to treat the spontaneous pneumothorax with intercostal drainage to underwater seal.

Under local anaesthesia a malecot catheter was introduced through the fifth space in the anterior axillary line, and when the catheter was connected to the underwater seal drainage no air bubbles came out. On attempting to adjust the position of the catheter, a strong tug synchronous with the heart beat was felt through the elastic catheter, and a more than average pull on the catheter failed to produce any change in its position. The patient's general condition remained unchanged, and P.A. and right lateral chest radiographs showed the catheter passing horizontally from the chest wall and blending with the collapsed lung shadow and the mediastinal shadow. It was felt that this represented a most unusual complication of the catheter piercing the collapsed lung and probably the pericardium. It was decided to carry out a right thoracotomy.

Through a right thoracotomy incision above the malecot stab wound portions of the fourth rib were removed, and the pleura was opened and found to be free of blood. The catheter was seen penetrating the pericardium. The pericardium was opened and contained no blood, and the catheter appeared to pass behind the superior vena cava into the space between it and the aortic arch. Attempts at withdrawal of the catheter showed the head was firmly fixed; it was pushed on a little and the head was cut off. The straight tube was withdrawn, and immediately a large haemorrhage from two holes in the superior vena cava occurred. One of the tears was controlled with a clamp, the other by the finger, while the incision was extended to divide the costal cartilages to provide better access. The superior vena cava was rotated and the internal wound sutured. Then the lateral wound was sutured and the clamp was removed. While these manœuvres were being carried out $96 \mathrm{oz}$. of blood was lost ; this was replaced by a transfusion. The lung was examined and a few bullae were seen at the apex but no obvious fistula. It expanded well. The pericardium was closed; the pleura closed and the wound was closed in layers.

Post-operative convalescence was uneventful and the patient was discharged home three weeks after admission with the lung fully expanded.

\section{DISCUSSION}

In attempts to reconstruct the sequence of events at the initial intubation two factors emerged.

One was that the trocar was inserted too far forward and inclined towards the mid-line so that when the tight-fitting catheter was pushed through the trocar it had crossed the anterior part of the pleura and met the superior vena cava without traversing the lung. Secondly, gross intrathoracic venous engorgement caused by breath-holding during introduction of the catheter through the trocar allowed the superior vena cava to be traversed by the taut malecot, and the expansion of the catheter on withdrawing the introducer sealed the puncture wounds in the vessel. This appears to be the only possible explanation. Attempts in the cadaver to push a malecot catheter on an introducer through the superior vena cava were unsuccessful no matter how much pressure was applied.

\section{CONCLUSION}

An unusual complication of intercostal intubation is described. It was the result of poor technique but in some respects the operative findings are difficult to explain. 